\title{
Assessment of Seismic Vulnerability of Reinforced Concrete Frame buildings
}

\author{
CHERIFI Fatiha ${ }^{1}$, FARSI Mohammed ${ }^{2}$, KACI Salah ${ }^{1}$ \\ ${ }^{1}$ University Mouloud MAMMERI, civil engineering department, Tizi-Ouzou, Algeria \\ ${ }^{2}$ National Center of Applied Research in Earthquake Engineering (CGS), Algiers, Algeria
}

\begin{abstract}
The seismic activity remains strong in the north of Algeria since no less than 30 earthquakes per month are recorded. The large number of structures built before the introduction of the seismic standards represents a high seismic risk. Analysis of damage suffered during the last earthquakes highlighted the vulnerability of the existing structures. In this study the seismic behavior of the existing buildings in TiziOuzou city, located in the north of Algeria, is investigated. To make this assessment, a database was created following a building inventory based on a set of technical folders and field visits. The listed buildings have been classified into different typologies. Only reinforced concrete frame buildings are considered in this paper. The approach adopted to estimate structures damage is based on four main steps: 1) construction of capacity curves using static nonlinear method "push-over", 2) estimate of seismic hazard, 3) determination of performance points, and finally 4) deduction of damage levels.
\end{abstract}

\section{Introduction}

Lors des tremblements de terre, les constructions subissent des oscillations horizontales, verticales et de torsion. Ces oscillations sont provoquées par des mouvements du sol d'assise qui imposent aux ouvrages des déformations. Contrairement aux oscillations verticales, les oscillations horizontales sont relativement mal supportées par les constructions, plus particulièrement lorsque celles-ci entrent en résonance avec le sol. Les oscillations de torsion, dues à la mauvaise conception des constructions, constituent un des phénomènes les plus destructeurs.

Lors des derniers séismes, il a été constaté que la quasi-totalité des effondrements, dans la ville de TiziOuzou, concerne les structures en portiques autostables en béton armé (BA) ; ce qui a causé d'importants dégâts matériels et des pertes en vies humaines. La vulnérabilité de ces structures est en effet élevée à cause de la mauvaise qualité des matériaux et de la mauvaise réalisation.

Cet article traite de la démarche adoptée pour la construction des courbes de capacité de ces structures par utilisation de la méthode push-over, la détermination des points de performance, et la déduction des niveaux de dommages induits par des scenarii préalablement définis.

\section{Typologie des bâtiments}

Cette typologie s'appuie sur des critères structuraux relatifs au comportement au séisme du bâtiment. La classification des constructions est effectuée selon quatre paramètres prépondérants: l'année de construction (niveau de conception parasismique), le système constructif, le matériau, ainsi que le nombre de niveaux. L'analyse de l'échantillon obtenu (données recueillies et enquête) a fourni le classement des typologies les plus représentatives (environ 94\%) du bâti de cette ville :

1. les maisons individuelles, de faibles hauteurs, constituées d'ossatures en béton armé, sans aucune conception parasismique (OBA0L : 46.54\%) ;

2. les bâtiments de hauteur moyenne, constitués d'ossatures en béton armé, construits sans conception parasismique (OBA0M : 18.98\%);

3 . les structures en voiles en béton armé, de hauteur moyenne, à faible niveau de conception parasismique (MBA1M : 10.48\%);

4. les structures en maçonnerie non renforcée, ne dépassant pas 02 niveaux (MNRL : 09.58\%) ;

5. les structures mixtes contreventées par des portiques

6. et des voiles en béton armé, de hauteur moyenne, sans conception parasismique (SM0M : 05.24\%) ;

7. les structures en voiles en béton armé, de hauteur moyenne, sans conception parasismique (MBA0M : $02.71 \%)$.

Notons que les premières règles parasismiques algériennes sont apparues en 1981 (RPA81). Ce 
règlement a connu des révisions successives durant les années 1983 (RPA81 version 83), 1988 (RPA88), 1999 (RPA99) et 2003 (RPA99 version 2003) suite au séisme de Boumerdes du 21 mai 2003. Ces dates, qui définissent les principales périodes d'évolution des règles parasismiques algériennes, correspondent à différents niveaux de conception parasismique.

Les principales caractéristiques du parc immobilier de cette ville sont illustrées sur les Figures (de 1 à 3). Pour mieux visualiser la répartition des différentes typologies sur toute la ville, les résultats sont présentés sur la carte de la Figure 4.

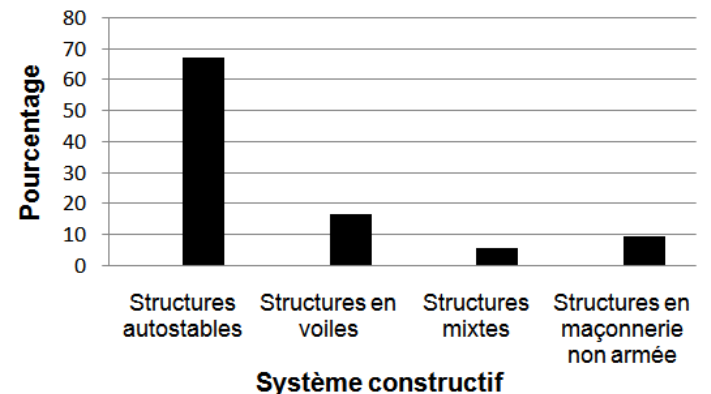

Fig.1. Classification selon le système constructif.

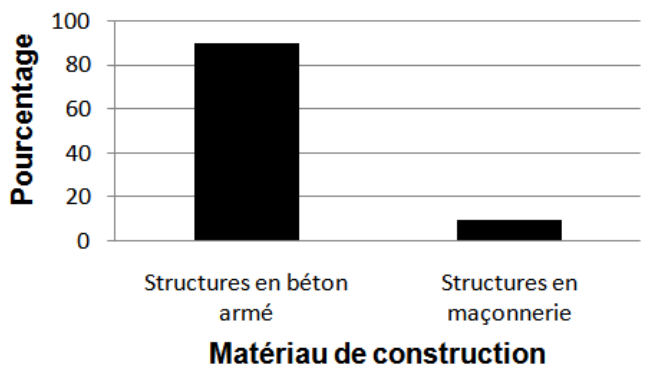

Fig. 2. Classification selon le matériau de construction.

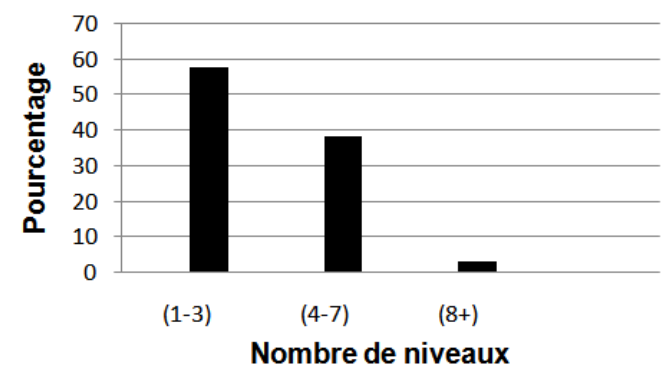

Fig. 3. Classification selon la hauteur du bâtiment.

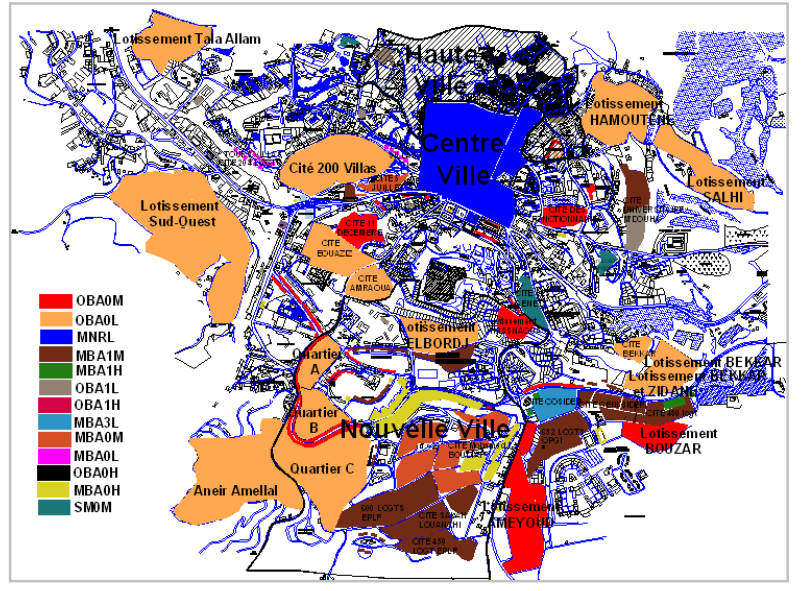

Fig. 4. Répartition des typologies les plus prédominantes de la ville de Tizi-Ouzou. Chaque couleur indique la typologie la plus prépondérante de la zone considérée. Seules les zones inventoriées sont illustrées sur la figure.

\section{Hypothèses de calcul}

Lors de l'analyse, la structure est considérée comme parfaitement encastrée à sa base, constituée de systèmes résistants reliés par des diaphragmes horizontaux rigides. Des rotules plastiques, introduites aux endroits où la plastification s'effectue de manière localisée, traduisent le comportement non linéaire des éléments. La courbe de capacité (effort à la base - déplacement au sommet) des structures est obtenue à partir d'un calcul statique non linéaire Push-over [1, 2].

L'état d'endommagement des rotules plastiques est fonction des niveaux de déformation [3]. Au niveau $I O$, les dommages structurels sont très limités, et la quasitotalité de la rigidité initiale est conservée. Au niveau $L S$, la section subit des dommages importants, pouvant entraîner une perte significative de sa rigidité. Le niveau $C P$ correspond à des dommages substantiels, au-delà desquelles la section est susceptible de se rompre. Notons que le point $B$ correspond à l'apparition de la première rotule plastique.

Les données nécessaires à cette analyse sont résumées dans le Tableau 1.

Tableau 1. Principales données utilisées dans les calculs.

\begin{tabular}{|l|l|}
\hline Types de données & Documents \\
\hline Dimensions des éléments & Plans \\
\hline Sections d'acier & Plans \\
\hline $\begin{array}{l}\text { Caractéristiques mécaniques } \\
\text { du béton }\end{array}$ & $\begin{array}{l}\text { Rapports } \\
\text { d'écrasement }\end{array}$ \\
\hline Caractéristiques des aciers & Notes de calcul \\
\hline Charges et surcharges & $\begin{array}{l}\text { Documents } \\
\text { techniques } \\
\text { réglementaires }\end{array}$ \\
\hline Caractéristiques du sol & Rapports \\
\hline
\end{tabular}




\section{Probabilités d'endommagement}

L'approche utilisée pour l'estimation des probabilités d'endommagement repose sur la méthode de capacité spectrale, qui se décompose en quatre étapes principales : la définition de la courbe de capacité, la définition du scénario sismique [4], l'obtention du point de performance (Fig. 5a), et la détermination des courbes de fragilité (Fig. 5b) [5].

Une fois les paramètres de la fonction de fragilité obtenus par la méthode HAZUS, l'on détermine et construit les courbes de fragilité par implémentation numérique de la méthode dans le logiciel MATLAB. Connaissant le déplacement maximal subi par un type de bâtiments, nous déduisons la probabilité d'atteindre ou de dépasser quatre niveaux de dommages (faible $S$, modéré $M$, important $E$ et très important $C$ ) (Fig. $5 \mathrm{c}$ ).

L'application de la méthode HAZUS nécessite que soit établie une correspondance entre les types de bâtiments considérés par cette méthode et les typologies définies pour la ville étudiée.

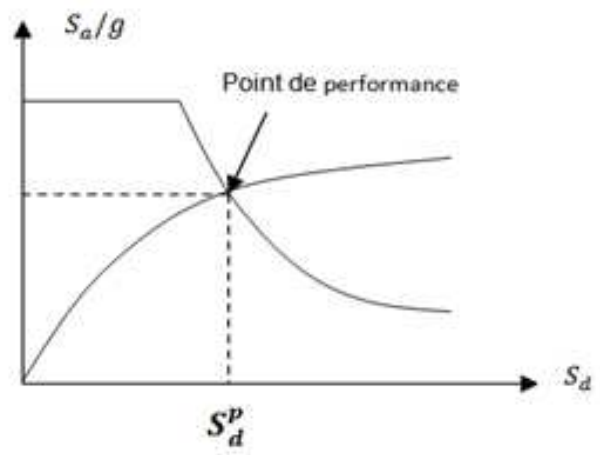

Fig. 5a. Définition du point de performance.

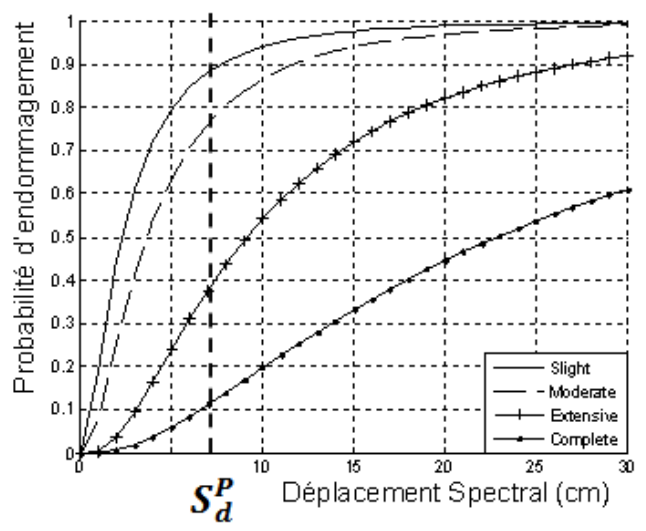

Fig. 5b. Courbes de fragilité selon la méthode HAZUS.

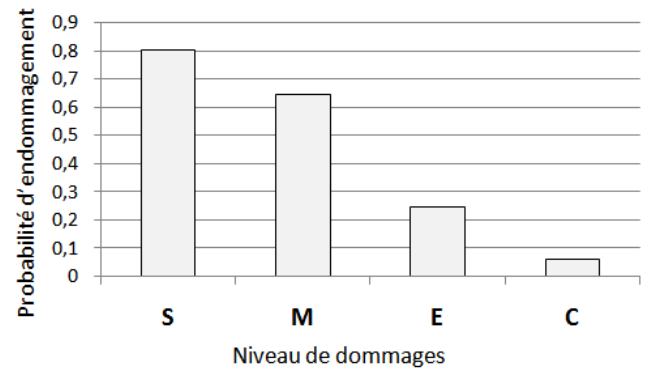

Fig. 5c. Estimation des probabilités d'endommagement.

\section{Résultats}

\subsection{Points de performance}

La méthode Push-over consiste à superposer la courbe de comportement de la structure (courbe de capacité) et la courbe de sollicitation du séisme (spectre de réponse) dans un même graphe de type (accélération spectrale $S_{a}$ déplacement spectral $S_{d}$ ). Dans ce plan, $S_{d}$ est le maximum en déplacement de la réponse du modèle équivalent à un degré de liberté qui reproduit le mode désiré, généralement le mode fondamental de vibration de la structure, et $S_{a}$ est le maximum en accélération de la réponse de ce même modèle.

L'intersection des deux courbes permet d'estimer le point de performance qui définit le déplacement maximal que peut avoir la structure, et donc de prédire les dommages induits. Les points de performance fournis par l'analyse Push-over, sont présentés dans les Tableaux (2 à 9). Pour chaque typologie, nous avons montré l'influence de la qualité du sol sur les points de performance et les niveaux de dommages y correspondant [6].

Tableau 2. Points de performance et niveaux de dommages Typologie OBA3L.

\begin{tabular}{|c|c|c|c|}
\hline \multirow[b]{2}{*}{ Type de sol } & \multicolumn{2}{|c|}{ Point de performance } & \multirow{2}{*}{$\begin{array}{l}\text { Type de } \\
\text { rotules } \\
\text { plastiques }\end{array}$} \\
\hline & $\begin{array}{l}\text { Déplacement } \\
D_{p}[\mathrm{~m}]\end{array}$ & $\begin{array}{l}\text { Force } \\
V_{p}[t]\end{array}$ & \\
\hline Rocheux & 0.048 & 225.57 & IO \\
\hline Ferme & 0.070 & 265.26 & IO \\
\hline Meuble & 0.087 & 279.84 & $\mathbf{L S}$ \\
\hline Très meuble & - & - & - \\
\hline
\end{tabular}

Table 3. Points de performance et niveaux de dommages Typologie OBA2L.

\begin{tabular}{|c|c|c|c|}
\hline \multirow[b]{2}{*}{ Type de sol } & \multicolumn{2}{|c|}{ Point de performance } & \multirow{2}{*}{$\begin{array}{l}\text { Type de } \\
\text { rotules } \\
\text { plastiques }\end{array}$} \\
\hline & $\begin{array}{c}\text { Déplacement } \\
D_{p}[m]\end{array}$ & $\begin{array}{l}\text { Force } \\
V_{p}[t]\end{array}$ & \\
\hline Rocheux & 0.019 & 74.68 & IO \\
\hline Ferme & 0.030 & 89.76 & LS \\
\hline Meuble & 0.038 & $97 . \overline{44}$ & LS \\
\hline Très meuble & - & - & - \\
\hline
\end{tabular}

Table 4. Points de performance et niveaux de dommages Typologie OBA1L.

\begin{tabular}{|c|c|c|c|}
\hline \multirow[b]{2}{*}{ Type de sol } & \multicolumn{2}{|c|}{ Point de performance } & \multirow{2}{*}{$\begin{array}{l}\text { Type de } \\
\text { rotules } \\
\text { plastiques }\end{array}$} \\
\hline & $\begin{array}{c}\text { Déplacement } \\
D_{p}[\mathrm{~m}]\end{array}$ & $\begin{array}{l}\text { Force } \\
V_{p}[t]\end{array}$ & \\
\hline Rocheux & 0.037 & 93.41 & IO \\
\hline Ferme & 0.059 & 105.45 & $\mathbf{L S}$ \\
\hline Meuble & - & - & - \\
\hline Très meuble & - & - & - \\
\hline
\end{tabular}




\section{Niveau de conception parasismique : faible}

Tableau 5. Points de performance et niveaux de dommages Typologie OBA0L.

\begin{tabular}{|c|c|c|c|}
\hline \multirow[b]{2}{*}{ Type de sol } & \multicolumn{2}{|c|}{ Point de performance } & \multirow{2}{*}{$\begin{array}{l}\text { Type de } \\
\text { rotules } \\
\text { plastiques }\end{array}$} \\
\hline & $\begin{array}{c}\text { Déplacement } \\
D_{p}[\mathrm{~m}]\end{array}$ & $\begin{array}{l}\text { Force } \\
V_{p}[t]\end{array}$ & \\
\hline Rocheux & 0.064 & 49.3 & IO \\
\hline Ferme & - & - & - \\
\hline Meuble & - & - & - \\
\hline Très meuble & - & - & - \\
\hline $\begin{array}{l}\text { Nombre de n } \\
\text { Niveau de co }\end{array}$ & $\begin{array}{l}\text { ux : (1-3) } \\
\text { ption paras }\end{array}$ & & \\
\hline
\end{tabular}

Table 6. Points de performance et niveaux de dommages Typologie OBA3M.

\begin{tabular}{|c|c|c|c|}
\hline \multirow[b]{2}{*}{ Type de sol } & \multicolumn{2}{|c|}{ Point de performance } & \multirow{2}{*}{$\begin{array}{l}\text { Type de } \\
\text { rotules } \\
\text { plastiques }\end{array}$} \\
\hline & $\begin{array}{c}\text { Déplacement } \\
D_{p}[\mathrm{~m}]\end{array}$ & $\begin{array}{l}\text { Force } \\
V_{p}[t]\end{array}$ & \\
\hline Rocheux & 0.051 & 150.34 & B \\
\hline Ferme & 0.078 & 192.53 & IO \\
\hline Meuble & 0.099 & 207.13 & $\mathbf{L S}$ \\
\hline Très meuble & - & - & - \\
\hline
\end{tabular}

Table 7. Points de performance et niveaux de dommages Typologie OBA2M.

\begin{tabular}{|c|c|c|c|}
\hline \multirow[b]{2}{*}{ Type de sol } & \multicolumn{2}{|c|}{ Point de performance } & \multirow{2}{*}{$\begin{array}{c}\text { Type de } \\
\text { rotules } \\
\text { plastiques }\end{array}$} \\
\hline & $\begin{array}{c}\text { Déplacement } \\
D_{p}[\mathrm{~m}]\end{array}$ & $\begin{array}{l}\text { Force } \\
V_{p}[t]\end{array}$ & \\
\hline Rocheux & 0.089 & 65.64 & B \\
\hline Ferme & 0.141 & 77.33 & IO \\
\hline Meuble & 0.188 & 83.75 & $\mathbf{L S}$ \\
\hline Très meuble & - & - & - \\
\hline
\end{tabular}

Table 8. Points de performance et niveaux de dommages Typologie OBA1M.

\begin{tabular}{|c|c|c|c|}
\hline \multirow[b]{2}{*}{ Type de sol } & \multicolumn{2}{|c|}{ Point de performance } & \multirow{2}{*}{$\begin{array}{l}\text { Type de } \\
\text { rotules } \\
\text { plastiques }\end{array}$} \\
\hline & $\begin{array}{c}\text { Déplacement } \\
D_{p}[\mathrm{~m}]\end{array}$ & $\begin{array}{l}\text { Force } \\
V_{p}[t]\end{array}$ & \\
\hline Rocheux & 0.037 & 82.83 & $\mathbf{B}$ \\
\hline Ferme & 0.058 & 109.42 & B \\
\hline Meuble & 0.071 & 121.48 & IO \\
\hline Très meuble & 0.116 & 134.31 & $\mathbf{L S}$ \\
\hline
\end{tabular}

Table 9. Points de performance et niveaux de dommages Typologie OBA0M.

\begin{tabular}{|c|c|c|c|}
\hline \multirow[b]{2}{*}{ Type de sol } & \multicolumn{2}{|c|}{ Point de performance } & \multirow{2}{*}{$\begin{array}{c}\text { Type de } \\
\text { rotules } \\
\text { plastiques }\end{array}$} \\
\hline & $\begin{array}{c}\text { Déplacement } \\
D_{p}[m]\end{array}$ & $\begin{array}{l}\text { Force } \\
V_{p}[t]\end{array}$ & \\
\hline Rocheux & 0.074 & 59.61 & IO \\
\hline
\end{tabular}

\begin{tabular}{|c|c|c|c|}
\hline Ferme & - & - & - \\
\hline Meuble & - & - & - \\
\hline Très meuble & - & - & - \\
\hline
\end{tabular}

\subsection{Dommages}

La probabilité d'atteindre ou de dépasser le niveau $C$, où les dommages sont très importants pouvant aller jusqu'à la ruine, atteint $6.0 \%$ pour les structures de faible hauteur et ne dépasse pas $10.5 \%$ pour les structures de moyenne et de grande hauteur. Ces valeurs sont considérablement réduites pour les bâtiments ayant un bon niveau de conception parasismique.

La probabilité de dépasser le niveau $E$, où les dommages sont importants, est inférieur à $25 \%$ pour les structures de faible hauteur et peut atteindre $40 \%$ pour le reste des typologies.

La probabilité de dépasser le niveau modéré $M$ de dommages atteint environ $88 \%$.

La probabilité de dépasser le niveau faible $S$ de dommages varie de $51 \%$ (typologies à bon niveau de conception parasismique) à plus de $97 \%$ (typologies à niveau moyen de conception parasismique).

\section{Conclusion}

Les principaux résultats obtenus suite à cette analyse montrent que les bâtiments construits après 2003 présentent une faible vulnérabilité. Les structures de faible niveau de conception parasismique perdent environ $50 \%$ de leurs rigidités initiales et subissent le maximum de dommages, pouvant entrainer l'instabilité et la ruine de la structure. Les courbes de capacité obtenues pour les structures construites avant 1981, sans aucune conception parasismique, sont nettement inférieures à la demande sismique et les points de performance sont inexistants. Ainsi, le déplacement engendré par le séisme est supérieur à la capacité de la structure. Il s'ensuit alors l'effondrement partiel ou total de ces structures.

Dans la plupart des structures construites avant 2003, les rotules plastiques apparaissent simultanément dans les poutres et dans les poteaux. L'état d'endommagement des rotules plastiques est plus avancé dans les poteaux et l'effondrement de la structure survient par rupture des poteaux des premiers niveaux. Notons que les sections de ces poteaux sont relativement faibles; ce qui peut induire une augmentation des efforts et des contraintes et par conséquent des déformations, conduisant à la ruine de ces éléments.

La ville de Tizi-Ouzou dans sa globalité présente une vulnérabilité sismique qui peut être qualifiée de faible pour les structures construites après 2003 et moyenne à importante pour les structures construites avant 2003. Des dégâts structuraux importants peuvent être subis 
notamment par les structures en ossatures en béton armé sans aucun niveau de conception parasismique.

\section{Références}

1. ATC-40, «Seismic Evaluation and Retrofit of Concrete Buildings », Applied Technology Council, Redwood City, California, (1996).

2. KRAWINKLER H., "Procedure and construction of pushover analysis of seismic performance evaluation engineering structure », vol: 20, edition: Elsever science, Department of civil engineering standford university, U.S.A, (1998).

3. Branson D. E., 《 Deformation of concrete structures》, New York (NY): McGraw-Hill, (1977).

4. D.T.R.-B-C.-2.48, «Règles parasismiques algériennes RPA99 version 2003 », C.G.S., Alger, (2003).

5. HAZUS, «Earthquake loss estimation methodology », Technical manuals, National Institute of Buildings Science, Federal Emergency Management Agency (FEMA), Washington, (1999).

6. CHERIFI F., FARSI M.-N., KACI S. «Évaluation de la vulnérabilité sismique des bâtiments existants en Algérie », Thèse de Doctorat en génie-civil, TiziOuzou, Algérie, (2016). 